\title{
Design of Crowdsourcing System for Analysis of Gravitational Flow Using X-ray Visualization
}

\author{
Ibrahim Jelliti \\ International Faculty of Engineering \\ Lodz University of Technology \\ Email: 200036@edu.p.lodz.pl
}

\author{
Andrzej Romanowski \\ Institute of Applied Computer Science \\ Lodz University of Technology \\ Email: androm@iis.p.lodz.pl
}

\author{
Krzysztof Grudzien \\ Institute of Applied Computer Science \\ Lodz University of Technology \\ Email: kgrudzi@iis.p.lodz.pl
}

\begin{abstract}
The paper describes application of the crowdsourcing system to pre-process $\mathrm{X}$-ray tomography images. In this paper, we show the analysis of the crowdsourcing system applied to process tomography imaging to investigate granular flow with aid of tracking particles. Applying crowdsourcing approach coupled with a proper system interface design enhances the performance of the workers and elevates the attitude. We show here how analysis of the proposed systems in terms of user adoption and performance. Proposed interface features are designed based on previous work evaluation in order to reduce the cognitive and physical workload demands.
\end{abstract}

\section{INTRODUCTION}

C ROWDSOURCING can be a delegation of work to be done, usually such that can be completed with Internet-connected computers [1]-[2], with an open call for people to contribute on an online task where the crowd here refers to an undefined, but aimed to be large, group of participants. Crowdsourcing systems coordinate those workloads to be completed by large groups of people to solve problems that a single individual could not achieve at the same scale, within the same budget or limited time, or a problem at high level of complexity. Another associated concept often revoked is the crowd intelligence that may result in either reaching the distinctive solutions to problems that are not so easily achievable for a single workers or is sometimes called power of averaging over large numbers of solutions to the same portion of work that leads to a perfect solution with no deviations form the correctly done work. Crowdsourcing system has been recently proved as an effective alternative to solve complex and mundane tasks [3] that can be solved by experts or as an alternative option may be distributed to several non-expert human operators that are willing to do the job or fragments of the job and therefore contribute to a joint solution of the problem. Sub-tasking the problem by the system typically lies to specific work flow depend on the problem, in dependent task, crowd system proves effectiveness in results while most of daily problems are more depend and much complex. Such task requires deep trained knowledge and experience that is difficult to formalize it in algorithms [4]. In crowdsourcing applications, contributors invited to accomplish system tasks using the human intelligence and capabilities [5]. During task submission, crowdsourcing serve as tool to gather information from the crowd intelligence. In the other side, the system relies on individual agent defined as expert serving for study and review the data gathered from the nonexperts and processed by the core system. This paper focus on developed crowdsourcing system that contributes nonexperts crowd for analysis if industrial tomographic images. The system introduce specific interface and features that work for enhance the crowd yield.

\section{RELATED WORK}

Crowdsourcing systems raised as a sort of mediation between the way computer systems process the surrounding world's parameters in comparison to how humans sense it. Most of tasks submitted to a widely available crowdsourcing servers (www.mturk.com, www.crowdflower.com, www.crowdmed.com) can possibly be processed by computer systems. Unfortunatelly due to a number of reasons such as difficulty of tasks, uniqueness, complexity, problems with achieving high accuracy or just the economical settings give rise to the need to use different methods for processing these datasets. Taking into account this work, especially image processing for research purposes is widely noted in [13]-[16]. Another interesting example of this type may be referred to a case of possible submission of the own medical results to the medial community around the world or in search for a diagnosis [17]-[19].

In this section, we review different examples of crowdsourcing applications, which demonstrate advantages of crowdsourcing aspects. Reviewing related work allows better to understand the aim of the paper concerning design and extensions of functionality dedicated to industrial process investigations.

\section{A. Crowdmapping}

In 2014, NASA launch a space research project 'Be a Martian' [6] based on crowdsourcing approach. The project invite people to mark the craters on mars and size it using bubble-marking interface that compute after user identify a mark. Similarly, The 'The Milki Way project' [7] is a research project that looks through tens of thousands of images from the 'Spitzer' Space Telescope. At the project website, crowd workers mark in specific platform galaxies, star clusters and space objects. The project give volunteers to enter the unknown or unusual things so they identify and size or telling what they see in the infrared data. After gathering 
all this information from crowds, it's easy to the system to classify and mining it.

\section{B. Crowdsourcing in Journalism}

Crowdsourcing is used in journalism to find story topics, information and sources. In recent years, a large amount of popular writing and relatively little academic research have been done in the areas of wisdom of the crowdsourcing, co-creation, and networked journalism. In 2009, the Guardian newspaper used crowdsourcing where readers (the crowd) were invited to investigate thousands of political documents that will help the newspaper to parse the data faster by CrowdSourcing. The online newspaper Huffington Post invited in 2009 their readers to compare the original stimulus bill from the US senate with the compromise. The volunteers asked to mark any the differences precisely identified examples of wasteful spending or corporate give ways that aren't stimulative.

\section{Crowdfunding}

Crowdfunding refers to funding a project or venture by raising monetary contributions from crowds online. Crowdfunding can be used in variety of purposes for instance Philanthropy and civic projects, Real estate crowdfunding, start-up investments, etc. At Kickstarter one of the famous platforms for crowdfunding, people gather funds for projects and ideas on various topics. In February 2012, a project named 'Double Fine Adventure' [8] collected over three million dollars to make a movie, in what was a recordbreaking crowdfunded project. In Crowdfunding, individual investments or donated funds typically are small and the funding power is based on the number of participants and whether investments or donated crowd-funding is an ideally way to quickly fund a project by this it can be better than the traditional funding models.

In this work we propose crowdsourcing system is dedicated to image processing of industrial processes and presents progress presented in comparison to system described in the [3]. As data processed in the system constitutes the image sequences, each person has at his disposal the ability to use not only the spatial information (based only on one image), but also temporal information. The presented system provides additional information to the current image, which is taken from previous images. The time relationship presented on the images is an added feature in comparison to previously presented crowdsourcing systems and should increase effectiveness of work.

\section{The CROWdSOURCING SySTEM}

The developed crowdsourcing system consists of several elements. Besides the data module, significant position is assigned to user interfaces, from customer (researcher/expert) and crowd point of view, also additional module dedicates to improve comfort of users work and thus obtain better results. Before describing crowdsourcing system, we present a short characteristic of X-ray measurement system with a deep explanation in interpretation tomography and radiography images to understand better the problem of analysis experimental data.

\section{A. X-ray tomography visualization}

The described system and the proposed approach to image processing and analysis is new solution in terms of industrial process investigations as the standard methods not always give sufficient information [20] - [25]. The images of gravitational flow in silo models were obtained with aid of $\mathrm{X}$ ray industrial tomography system [10] - [11]. Data recorded from X-ray system can be presented in form of 3D images (see fig. 1). However for this kind of data visualization is necessary to stop flow process, in analyzed case stopping of gravitational flow of solid, and rotated silo in space between the source and X-ray detector to have the whole set of projections. The 3D image is result of reconstruction procedure based on 2D radiography images (projections). But such approach, for flow phenomena investigation, forces stopping the flow what can be reason of flow characteristic changes - invasion in process. The better option is to apply the continuous record of data. This solution was used during experiments and results were saved in form of $2 \mathrm{D}$ radiography images, an example of single raw radiography is presented in figure 2. Quality of this visualization is lower than the case of 3D images (see fig. 1), what causes more complication in image processing and analysis procedure. The quality of radiography images are resulted of absorption of X-ray radiation by all material located on line between source of radiation and a single pixels on X-ray detector. In presented investigation was used 2D flat panel detector. The level of signal detected in a pixel, is due to absorption of X-ray radiation by material structure on path between source and detector pixel. The radiography image provides information about average value of absorption level, which is directly related with material concentration. More details about application of X-ray system to gravitational flow of solid can be find in [10].

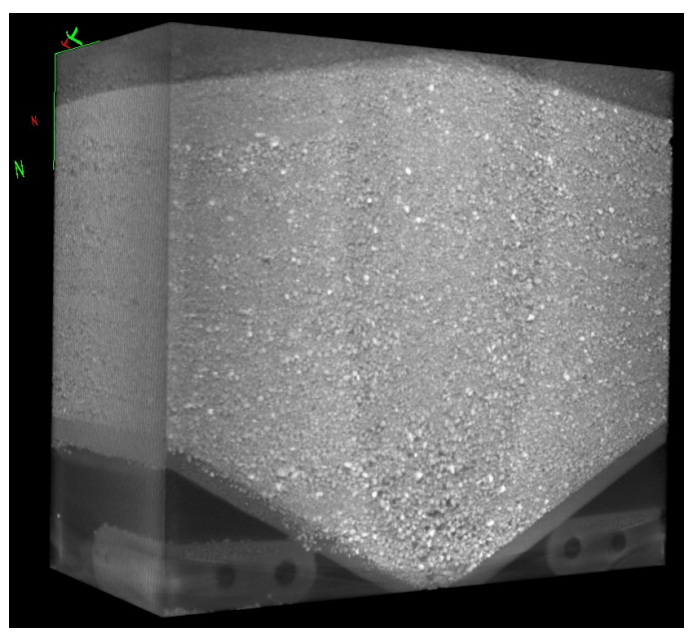

Fig. 1. 3D X-ray tomography of gravitational flow in silo 


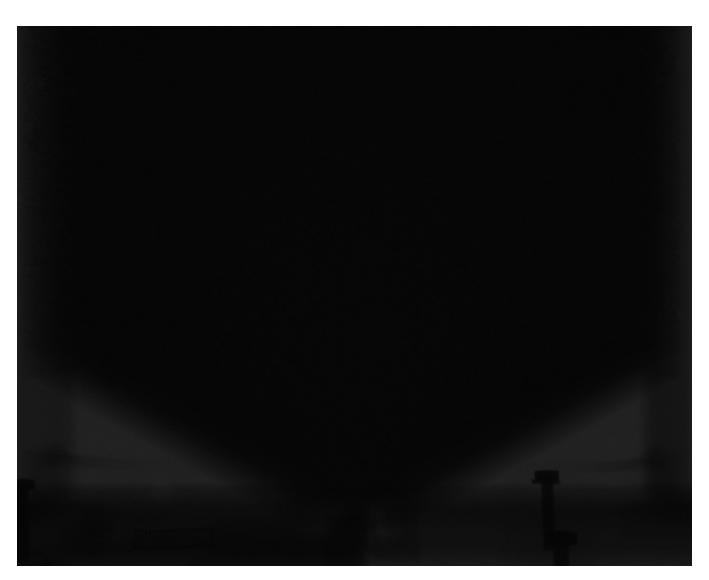

Fig. 2. RAW X-ray radiography image

After continuously recorded flow in sequence of $2 \mathrm{D}$ radiography images was applied correction of the tomographic images. The result of this processing is presented in figure 3, radiography image for stopped flow, and in figure 4, radiography image for continuously flow. Further improvement based on normalization procedure allows to better visualize center region of silo [11]. Figure 5 presents an example of a normalized radiography image.

As was mentioned the radiography images provide information about the material concentration distribution. On the presented images (see fig. 3-5) it's visible the different area of granular material with different level of concentration. Such flow phenomena is characteristic for funnel flow [9], where in the center of silo material is at lower concentration than at silo wall - stagnant zone appear. The distinguish of these two area is much easier to noticed in 3D tomography (see fig. 2) than in 2D radiography image (see fig. 3-5). Non moving material in silo allows to obtain better radiography image (see fig. 3) than for moving material (see fig. 4). But the influence of breaking flow on process causes different granular behavior than for free flow.

The main task for flow investigation was analyzed dynamic of gravitational flow in funnel area. In order to achieve the intended task the tracking particles, with higher X-ray absorption were added to granular material. The algorithm of image analyzes should provide trajectories of each single particles. It is not easy task to find all tracking particles, What is visible on figures (see. fig. 3-5). The results of automatic image processing algorithm doesn't provides full information about all particles [10]. The development of the crowdsourcing system should allow to find more particles in sequence of radiography images and complete knowledge about flow.

\section{B. System workflow}

The system is based on user oriented interface. First, the experts identify the task to accomplish and take series of $\mathrm{X}$-ray images of the silos. Then, the obtained data will processed for correction and optimization.

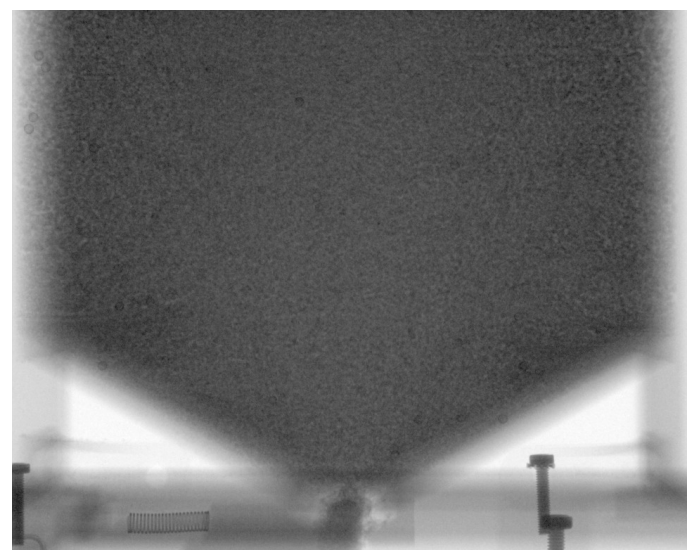

Fig. 3. Radiography image with stopped granular flow

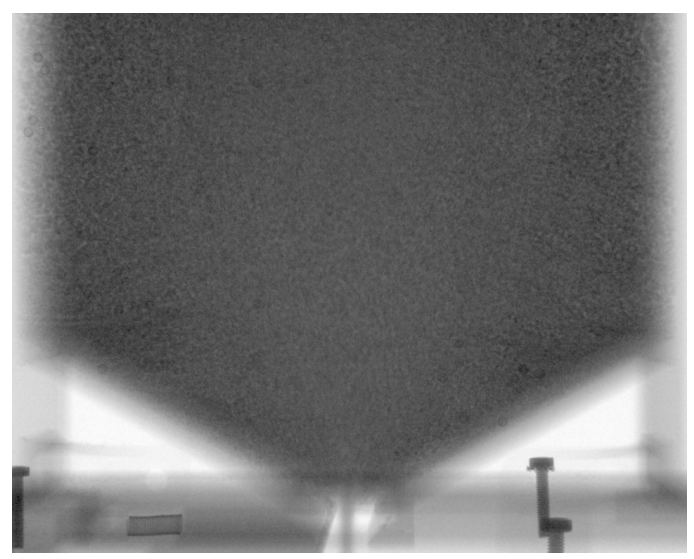

Fig. 4. Radiography image with free granular flow

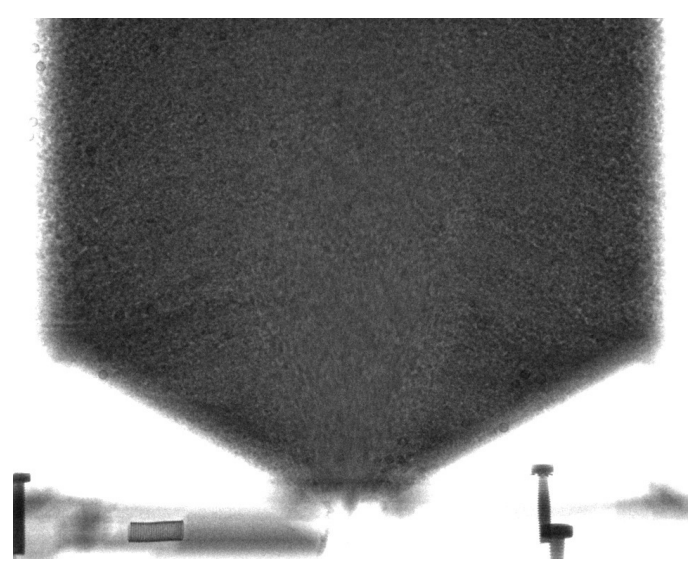

Fig. 5. Radiography image after normalization procedure

The system workflow runs in three steps, first experts how prepare the x-ray data (in this paper there are radiography images) upload them using the system core into the database. Then the system core can generates tasks from the uploaded data whenever any person of the crowd access to the tasks webform. The system core here select set of data in the database that is not submitted yet by the person, from these 
collection, tasks are created in form of 7 to 25 images per task in random way. The creation of task is happen at crowd workers side that means every person of the crowd has his own tasks display. At the third step, the worker chooses a specific task the core system task the task frames into specific interface for analysis of frames. Once worker launch his work submission the system core collect the frames mark data in structured form and save it into the database for later data mining.

\section{System interface}

Each workers' crowd member can browse the automatically generated task with information about the number of frames like presented on figure 6 , then he could choose the one to accomplish. The system redirects the worker to an appropriate interface where all task frames are presented in slide show display together with variety of tools developed to enhance the task performance in time and quality meaning.
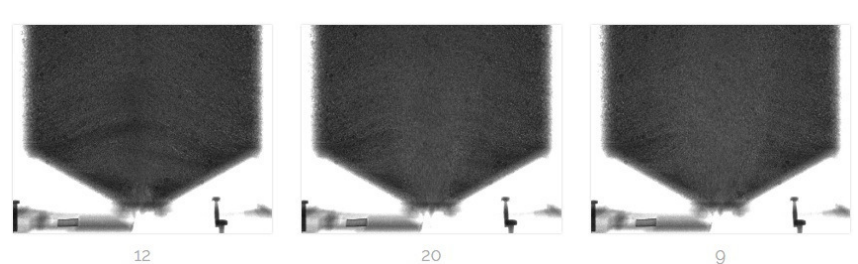

Fig. 6. Tasks interface

\section{Task web interface}

Once the worker chooses a task to accomplish automatically we be redirected to the another web-interface dedicated to analysis of the tomographic image. The new interface informs the worker at the very begin about the next interface component, features available to help him in doing the task and timing count for his work per frame in background. This interface is presented in figure 7. Such tool panel on the right-hand side is well known in other domains of crowdsourcing applications, but has not been reported for analysis of research tomography images yet.

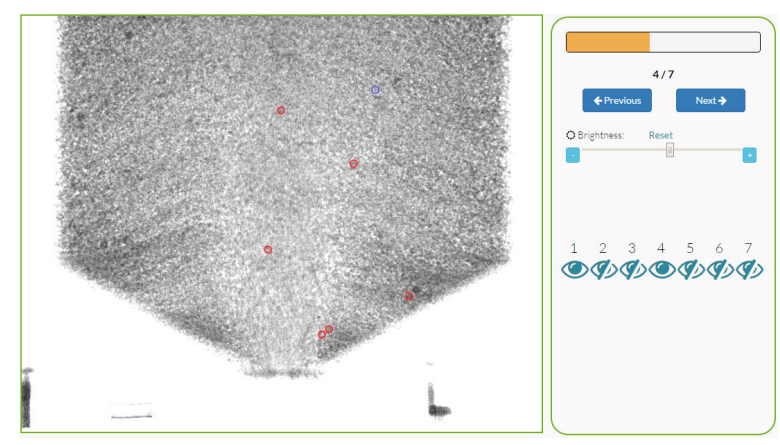

Fig. 7. System interface

\section{E. System Features}

1) Interface interactivity: In each frame, worker has to mark with double-click each particle that appears to him in the current frame.

- Double-click on mark will remove it from the frame.

- Single-click on mark will result to be selected.

- Moving the mouse on keep the mouse-key down will let the particle to move according the mouse coordinate in the frame.

2) Frame adjustment: Furthermore, the interface offer on the right-hand panel more features to adjust and ease the job of visual working on the tasks.

First a workload progress bar on the top right shows the progress of the job. Next, a frame caption below shows the index of current frame over the total number of processed frames. The user can browse between the frames easily while doing the task so he can learn from the changes in frames to identify the mark. The interface offer an image processing functions to adjust the current frame. The adjustment of frame brightness help the user to recognize better a particle inside the silo that was not well visible for him in the regularly displayed $\mathrm{x}$-ray frame (as shown on figure 8).

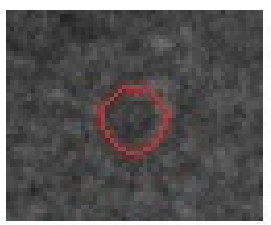

before

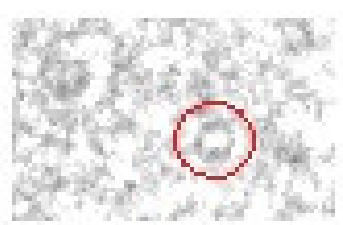

\section{After}

Fig. 8. Brightness adjustment effect on area of the frame.

3) Particles visualization: The system has an 'eye-bar' appear below the brightness control allows the worker to review his particle trace of previous frame on the current frame (reviewing by hide and show the others frame particles). this feature work for increasing the performance of the worker in the frame.

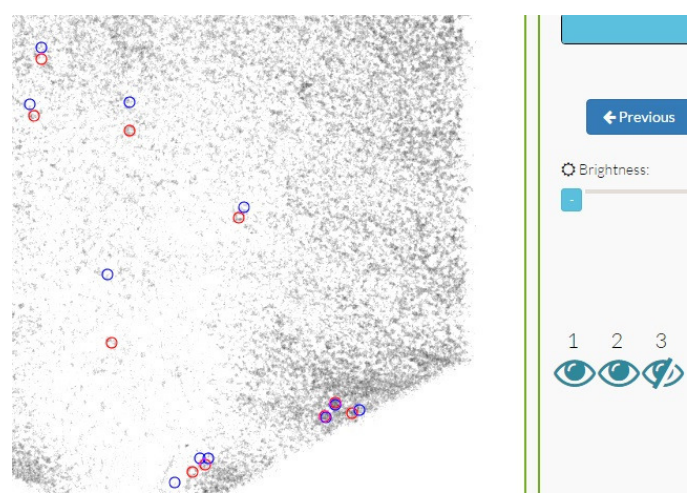

Fig. 9. Worker at frame 2 made mark in red and seeing the marks from previous frame in blue in the same frame. 


\section{PRE-STUDY \& RESUlTS}

System was firstly tested and evaluated by experts ( 2 people with skills in system design, X-ray image analysis, flow process analysis knowledge and previous experience with scientific images crowdsourcing systems). Experts prepared content of the test for the regular users' study. During the study and the evaluation on the system, we conducted tests with 7 non-expert workers completing the tasks. The workers were asked to accomplish task of 41 frames. Chosen frames were a fragment of a time series of X-ray consecutive radiography images and were specially selected to constitute a relatively difficult dataset in terms of marking trace particles. The results are archived by the system after every frame submitted by the workers. Besides the regular study goals, we also inquired workers about their experience in different aspects of using the system and this feedback help us in qualitative evaluation of the system performance.

\section{A. System-side results}

Since the main goal of the system is to enable correct identification of the trace particles the proposed design aimed at supporting to achieve this goal. Figure 10 shows an example of results where plots of different colors indicate the number of particles marked by the users for consecutive frames. These results were obtained only with possibility to change the brightness (no temporal hints). One can see a specific spread of counted values, however experts assessed these results as acceptable provided the quantitative analysis (visual inspection) shows that missed particles are not significant regarding the domain analysis. Including the temporal factor into the workflow, i.e. enabling users to use previous frames' results as well as possibility to browse frames back and forth significantly reduced the spread of particles count for different workers. The min difference between expert results and crowd study results decreased from $7 \%$ to $1 \%$ while max difference decreased from $24 \%$ to $9 \%$ and in general lead to equalize the results of the crowd, approaching the number expected by experts.

Figure 11 shows the marked particles pointed out by the crowd on the images. The particle easiest to find, located at silo wall, are marked by all workers. The main problem is the discrepancy visible in the funnel area of the flow (central zone of the image). However, when using the temporal options of the interface results tend to the similar homogeneity as for the by-the-walls zones that proves our concept of using additional information in order to ease the process of finding the particles and increasing the accuracy of the system.

In this situation, the system applies basic algorithm to compute real number of particles. In order to do that system considers a particle where most of users marked circles close to anothers' marks by applying an unsupervised clustering at high inner criteria measurements.

\section{B. Human-side results}

The other type of analysis we did concerns the crowd workers performance but this time it is associated with the

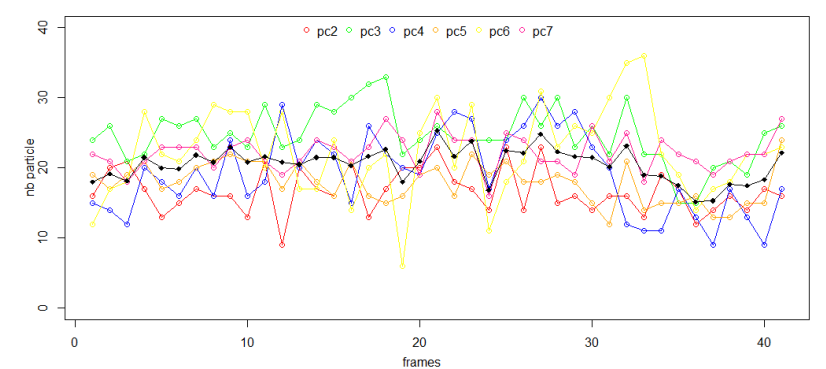

Fig. 10. Plot of numbers of particles marked by users on consecutive images.

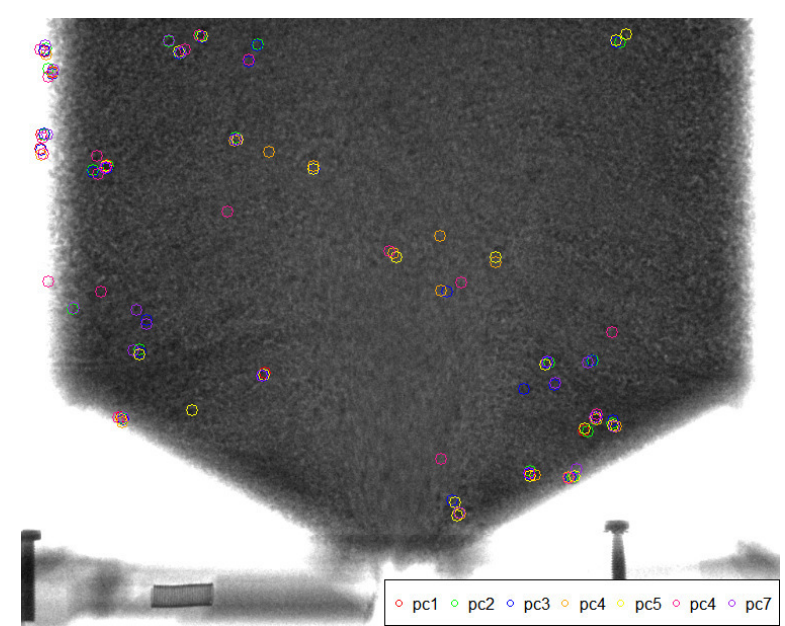

Fig. 11. Image showing particles marked by different users superimposed on a single frame.

other factors of humans' work disregarding the numerical results of particle counted. The analysis of these additional parameters is expected to give insight for further system development. One of the core modules is the time meter recording the period spent by the worker on each frame within the task. It may give some information about the difficulty of the task (or a given frame; especially if the particle count of the different workers for this frame has a large spread of values) but it require a longer study and more participants to derive specific conclusions about that. The system notifies the worker about timer counting per frame before launch the task. The timer feature count time per frame with possibility of browsing the collection back and forth and this enables to measure the performance of the system for the crowd in different frames. Figure 12 shows a heat map of a single experiment where each column represents time performance of a single worker in time from the bottom up to the top for all 41 frames. Yellowish colors indicate more time spent on a single frame while navy blue corresponds to shorter processing of a single frame. After experiments a survey was conducted. Workers answered 8 questions related to task demands and system features. Figure 13 shows a subjective performance in a perceived sense of time users felt themselves in completing the task.

Workers were also asked about the perceived workload in 


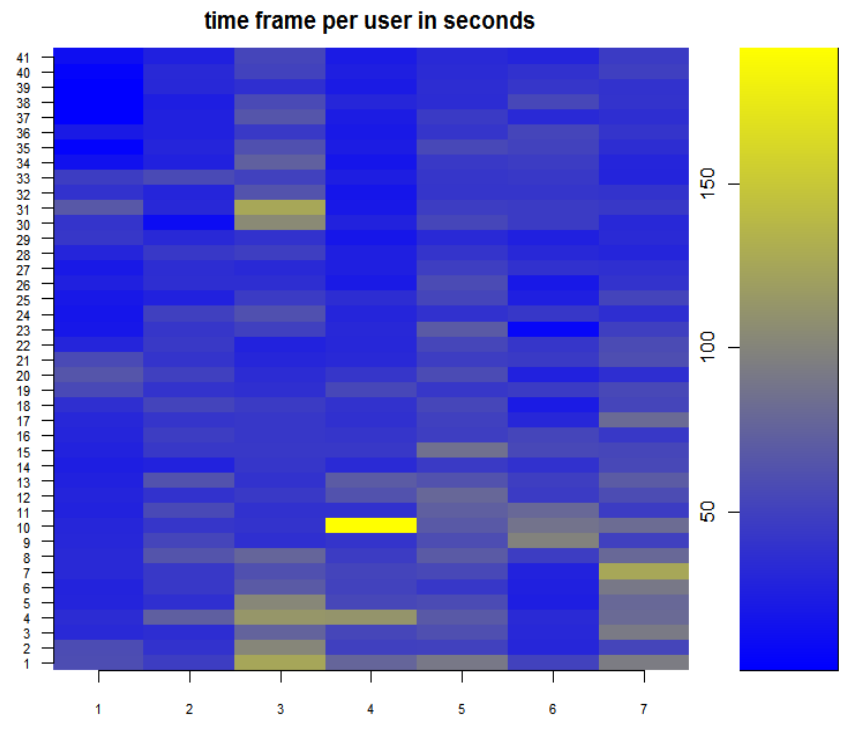

Fig. 12. Work-time per frame for all user

Performance Demand

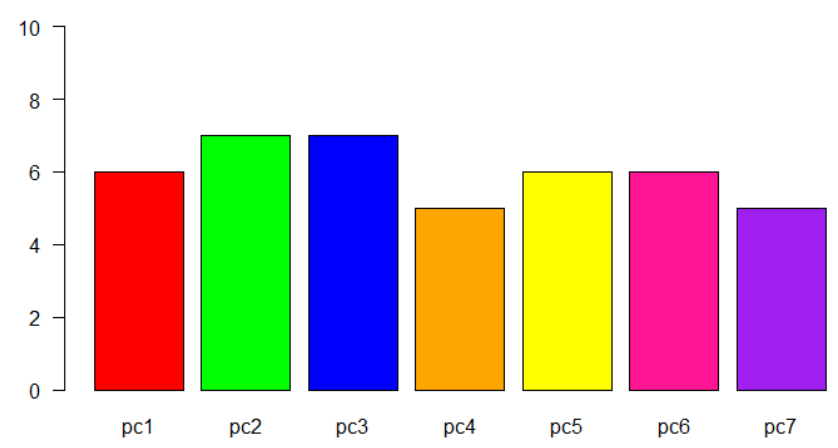

Fig. 13. Crowd workers perceived self-performance.

terms of effort, frustration level, learning, mental, temporal and physical aspects during accomplishing the task. Results are given on figure 14 .

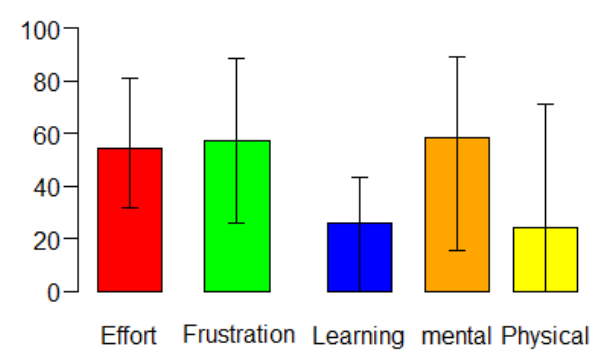

Fig. 14. Results on subjective crowd workers' feedback about perceived workload during the study.

The workers feedback reflect the computation advantage in realizing, the learning level is $20 \%$ as average and reach zero even. This indirectly means that the majority of workers do not browse the collection of frames to learn how to identify the particle. This is interesting since the flexibility of the interface was a very demanded feature by the experts. The conclusion for future is to take a deeper look into this aspect. One of the possibility is that the initial training of the users before the experiment/task has to be more detailed.

The level of physical demand is nearly the same this back to the efficacy of the features in the system interface. We have asked the crowd how much the interaction with mouse and brightness control was helpful in accomplishing the frame analysis. The results are promising as presented on figure 15.

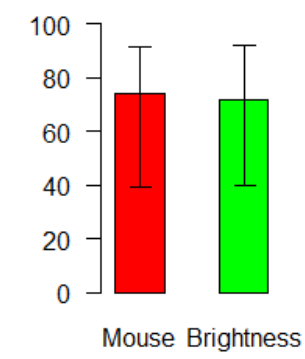

Fig. 15. Crowd workers feedback on system features.

\section{Discussion \& Future WORK}

When looking a the results for a system and humans as interpreted in the previous section we noticed a significant level of effort demand against the physical demand beside similar level of frustration. This a general and widely known problem concerning repetitive tasks and can be explained in a way that worker marks the particles in the frame and the same for the rest of collection even with the eye bar feature that reduces at him computing mark again and it may get higher harder if the choose a large collection to work. To enhance the system framework for better feedback results, we work on changing the manner how the work must perform to accomplish a task, so we may aim to decrease the effort by mouse clicking with automatically displaying the previous frame marks to the current and the worker will have just to drag the copy-particle to their new position. However, the previous experience of the experts reveal that this solution may be perceived as distraction for some workers. Therefore, flexibility of the interface in this aspect is required.

In order to cope with high complexity during the clustering of marked particles future work on parallelisation of trajectory calculation with the work of crowdworkers at the same time when he mark the particles may be considered.

Finally, we work on technique that tight the visual search space for the worker. We expect that the implication of this technique in the crowdsourcing system will result in decreasing the effort, mental, physical and temporal demand and at the same time hopefully increase the feasibility and scalability of the crowd system. Heading for these improvements will lead us to semi-automated crowd system that may be comparable with possible automatic algorithms in this complex problem. It seems to be interesting to utilize the eye tracking system to find out how worker use 
crowd system [26]. Results will allow to improve the system from worker point of view. Similarly hands gesticulation recognition can be very useful to explore new direction of human computer interaction development in crowdsourcing based system [12].

\section{CONCLUSion}

In this research study we analyzed the design aspects of crowdsourcing system developed for the investigation of gravitational flow with aid of X-ray images. The results are presented on the background of the distinct elements of system design. The additionally introduced features of brightness and especially a feature allowing to use the temporal information, i.e. browsing frames of the current task back and forth allowed to obtain more stable results; less diverse distribution of results delivered by different workers was observed. From the further research on the scientific crowdsourcing systems point of view, the presented heat map is a valuable tool for quantitative estimation of workload per frame since it enables analysis of time spent as well as another valuable information about how often workers used back and forth option. The designed system allows to generate dynamic tasks at worker-side. With the task interface features, we get satisfactory results from the experiments that build on the earlier published work for similar application. The crowd study on the system shows the system feasibility in solving the target problem and improvement in some aspects of the interface performance over the previous design for investigation of industrial flow processes.

\section{ACKNOWLEDGMENTS}

This work is partially funded by the European Commission under the Erasmus Mundus E-GOV-TN project (Open Government data in Tunisia for service innovation and transparency) -EMA2; Grant Agreement no. 2013-2434/001001. Authors would like to acknowledge inspiring previous work conducted by all co-authors of [3].

\section{REFERENCES}

[1] Brabham, D. C., "Crowdsourcing as a Model for Problem Solving. an introduction and cases", Convergence: The International Journal of Research into New Media Technologies 14(1), pp. 75-90, 2008.

[2] Howe J., Crowdsourcing: why the power of the crowd is driving the future of business, New York: Crown Business, 2008.

[3] Chen C., Wozniak P., Romanowski A., Obaid M., Jaworski T., Kucharski J., Grudzien K., Zhao S., Fjeld M., "Using Crowdsourcing for Scientific Analysis of Industrial Tomographic Images", ACM Transactions on Intelligent Systems and Technology (TIST),Vol. 7, 4, Article 52, 2016, DOI: http://dx.doi.org/10.1145/2897370, 26p.

[4] Stol, K.J. and Fitzgerald, B. Researching, "Crowdsourcing software development: perspectives and concerns", ICSE14 Workshop on Crowdsourcing in Software Engineering, pp. 7-10, 2014.

[5] G. Little, L. Chilton, M. Goldman and R.C. Miller, "Exploring iterative and parallel human computation processes", HCOMP '10 Proceedings of the ACM SIGKDD Workshop on Human Computation, pp. 68-76, 2010.

[6] NASA Citizen Science Lab: beamartian.jpl.nasa.gov/welcome.

[7] ww.milkywayproject.org the Milky Way project news: www.jpl.nasa. gov/news/news.cfm?release $=2012-062$

[8] www.kickstarter.com/projects/doublefine/double-fine-adventure.
[9] D. Schulze, Powders and Bulk Solids: Behavior, Characterization, Storage and Flow, Springer, Verlag, Berlin, GmbH Co. K., 512 p., 2008

[10] K. Grudzien and M H. de la Torre Gonzalez, "Detection of tracer particles in tomography images for analysis of gravitational flow in silo", Image Processing and Communications, Vol. 18, pp. 11-22, 2013.

[11] L. Babout, K. Grudzien, E. Maire, and PJ. Withers, "Influence of wall roughness and packing density on stagnant zone formation during funnel flow discharge from a silo: An X-ray imaging study", Chemical Engineering Science, 97, pp. 210224, 2013.

[12] Półrola M., Wojciechowski A., "Real-Time Hand Pose Estimation Using Classifiers", Computer Vision and Graphics: International Conference, ICCVG 2012, Warsaw, Springer Berlin Heidelberg, pp. 573$580,2012$.

[13] Irshad H., Montaser-Kouhsari L., Waltz G., Bucur O., Nowak J.A., Dong F., Knoblauch N.W. and Beck A. H., âĂIJCrowdsourcing image annotation for nucleus detection and segmentation in computational pathology: evaluating experts, automated methods, and the crowdâĂİ, Pacific Symposium on Biocomputing, pp.294-305, 2015.

[14] Deepti Ghadiyaram, Alan C. Bovik, "Crowdsourced study of subjective image quality", 48th Asilomar Conference on Signals, Systems and Computers, pp. 84-88, IEEE, 2014

[15] Cabezas F., Carlier A., Salvador A., Giro-i-Nieto X., Charvillat V., "Quality Control in Crowdsourced Object Segmentation", IEEE International Conference on Image Processing (ICIP), Quebec City, Human-Computer Interaction, 2015, DOI: 10.1109/ICIP.2015.7351606.

[16] Flavio Ribeiro, Dinei Florencio ; Vitor Nascimento, "Crowdsourcing subjective image quality evaluation", 2011 18th IEEE International Conference on Image Processing, pp. 3097-3100, 10.1109/ICIP.2011.6116320.

[17] Max H Sims, Maria Fagnano, Jill S Halterman, Marc W Halterman, "Provider impressions of the use of a mobile crowdsourcing app in medical practice", Health Informatics Journal August 28, 2014.

[18] Meyer A.N.D; , Christopher A Longhurst, Hardeep Singh, "Crowdsourcing Diagnosis for Patients With Undiagnosed Illnesses: An Evaluation of CrowdMed", Journal Of Medical Internet Research, vol 18, No 1, 2016.

[19] Liliya I. Besaleva, CrowdHelp: "A crowdsourcing application for improving disaster management", Global Humanitarian Technology Conference (GHTC), IEEE, pp. 185-190, 2013.

[20] Romanowski, A.; Grudzien, K.; Chaniecki, Z.; Wozniak, P., "Contextual processing of ECT measurement information towards detection of process emergency states", 13th International Conference on Hybrid Intelligent Systems (HIS), TUNISIA, IEEE, pp. 291 - 297, 2013

[21] Romanowski, A.; Grudzien, K.; Sankowski, D.; Aykroyd, R.G.; Williams R.A., "Advanced statistical computing for capacitance tomography as a monitoring and control tool", 15th International Conference on Intelligent Systems Design and Applications (ISDA 2005), Wroclaw, Poland, pp. 49-54, 2005.

[22] Wajman, R.; Fiderek, P.; Fidos, H.; Nowakowski, J.; Sankowski, D.; Banasiak, R., "Metrological evaluation of 3D electrical capacitance tomography measurement system for two-phase flow fraction determination", Measurement Science and Technology, vol. 24, no. 6, pp. $1-11,2013$

[23] Mosorow, W., "Flow Pattern Tracing for Mass Flow Rate Measurement in Pneumatic Conveying Using Twin Plane Electrical Capacitance Tomography", Particle and Particle Systems Characterization, vol. 25 , no. 3 , pp. 259-265, 2008.

[24] Sikora, J., Panczyk, M., Wieleba, P., "Hybrid boundary element method applied for diffusion tomography problems", in: Computer Vision in Robotics and Industrial Applications, (Series in Computer Vision); Eds. Nowakowski J., Sankowski D. - [Hackensack] New Jersey: World Scientific Publishing, pp. 197-229, 2014

[25] Kapusta, P., Majchrowicz, M., Sankowski, D., Jackowska-Strumiłło, L., Banasiak, R., "Distributed multi-node, multi-GPU, heterogeneous system for 3D image reconstruction in Electrical Capacitance Tomography - network performance and application analysis, Przegląd Elektrotechniczny, 89 (2 B), 2013, pp. 339-342.

[26] Wojciechowski A., Fornalczyk K., "Single web camera robust interactive eye-gaze tracking method", Bulletin of the Polish Academy of Sciences Technical Sciences, vol. 63, no. 4, pp. 879-886, 2015. DOI: 10.1515/bpasts-2015-0100. 